\title{
Low-Temperature Protein Dynamics Studied by the Long-Lived Stimulated Photon Echo
}

\author{
Daniël Thorn Leeson, Otto Berg, and Douwe A. Wiersma* \\ Ultrafast Laser and Spectroscopy Laboratory, Department of Chemistry and Materials Science Centre, \\ University of Groningen, Nijenborgh 4, 9747 AG, Groningen, The Netherlands
}

Received: December 6,1993

\begin{abstract}
The spectral diffusion of $\mathrm{Zn}$-substituted cytochrome $c$ in a glycerol/water host glass has been investigated on a time scale from picoseconds to milliseconds using photon echoes. The results are interpreted within the two-level system model and by using the concept of conformational protein substates. The experiments reveal a gap in the distribution function of two-level system relaxation rates between $1 \mathrm{MHz}$ and $10 \mathrm{kHz}$. Evidence is provided for the existence of two distinct types of two-level systems.
\end{abstract}

\section{Introduction}

Proteins exhibit conformational fluctuations around a mean tertiary structure. ${ }^{1}$ The microscopic disorder of proteins is often described using the concept of conformational substates. ${ }^{2}$ These substates arise from atoms in certain regions of the protein residing in two or more metastable positions. The substates may be separated by a wide distribution of conformational barriers. This view is equivalent to the two-level system (TLS) model ${ }^{3,4}$ that has succesfully accounted for many of the anomalous properties of glasses.

It is becoming clear that optical line-narrowing phenomena can play a key role in determining to what extent proteins resemble glasses and whether theoretical models that have been proposed for the description of glasses, such as the TLS model, are valid for proteins as well. Line-narrowing techniques such as photon echoes and hole burning have proven to be excellent tools for the study of glass dynamics [for a review see ref 5]. Generally speaking, these techniques study the optical dynamics of impurity molecules in a host material. The optical absorption spectrum of an ensemble of chromophores embedded in an amorphous host is often inhomogeneously broadened due to inhomogeneieties in local strain fields or electric fields experienced by individual chromophores. When the host is a glass or a protein, the inhomogeneous line width is several orders of magnitude larger than the homogeneous line width. The structural disorder that causes the inhomogeneous broadening is often not static but changes with time. Therefore, the transition frequency of a single chromophore is a dynamical property. This phenomenon is commonly referred to as spectral diffusion. Due to spectral diffusion, the effective homogeneous line width as measured in a photon echo or hole-burning experiment depends on the time scale of observation. Spectral diffusion can occur in proteins because of relaxation of the individual substates.

The past few years have seen a steady increase in the use of optical line-narrowing techniques to study protein dynamics. Boxer et al. ${ }^{6}$ performed nonphotochemical hole burning on $\mathrm{Zn}$ pyrochlorophyllide $a$-myoglobin embedded in a poly(vinyl alcohol) film. Both the holewidth and its temperature dependence were comparable with $\mathrm{Zn}$-pyrochlorophyllide $a$ esterified with 3-(3'-hydroxypropyl)pyridine in a polystyrene film. Koloczek et al. ${ }^{7}$ showed that the fluorescence line-narrowing spectrum of $\mathrm{Zn}$ cytochrome $c(\mathrm{Zn}$-cyt $c)$ in a glycerol/water host at $10 \mathrm{~K}$ closely resembles that of $\mathrm{Zn}$ mesoporphyrin IX (Zn-MP) in a diethyl ether matrix. They concluded that the chromophores experience a continuous distribution of sites in the protein. Zollfrank et al. ${ }^{8}$ used temperature cycling photochemical hole burning to study spectral diffusion of mesoporphyrin IX (MP) in horseradish

- Abstract published in Advance ACS Abstracts, March 1, 1994. peroxidase in a glycerol/water host. One of their conclusions was that structural relaxation of the protein takes place at temperatures as low as $10 \mathrm{~K}$. They observed a steplike broadening of photochemical holes as a function of the magnitude of the temperature excursion. This behavior was found to be clearly different from that of the MP-glycerol/water system. ${ }^{9}$ These results were interpreted in terms of a lower density of TLSs in the protein. It was argued that only a single TLS is active. Similar conclusions were drawn by Saikan et al. ${ }^{10}$ on the basis of nonexponential incoherent photon echo decays of iron-free myoglobin below $10 \mathrm{~K}$. Recently, Gafert et al. ${ }^{11}$ studied the same system by pressure tuning hole burning at $1.5 \mathrm{~K}$. They found remarkable differences between the behavior of protoporphyrin IX (PP) in myoglobin in a glycerol/water host and that of PP directly dissolved in a glycerol/DMF glass.

Here, we report on a time-resolved study of the spectral diffusion of $\mathrm{Zn}$-cyt $c$ in a glycerol/water matrix at $1.8 \mathrm{~K}$, making use of the long-lived stimulated photon echo technique. ${ }^{12}$ Also, twopulse photon echoes (2PEs) were measured. The results are directly comparable to recent studies of spectral diffusion in doped amorphous solids, performed in our laboratory. ${ }^{13,14}$

\section{Experimental Section}

Cytochrome $c$ from horse heart was obtained from Sigma. $\mathrm{Zn}$-cyt $c$ was prepared using the method of Vanderkooi et al. ${ }^{15}$ The protein was dissolved in $10 \mathrm{mM} \mathrm{PO}_{4}$ buffer (pH 7.0) after which the volume was quadrupled by the addition of glycerol. Samples were degassed and sealed in a $1-\mathrm{mm}$ optical path-length cuvette. The optical absorption spectrum at $2.0 \mathrm{~K}$ is shown in Figure 1. The glycerol/water host glass was prepared by plunging the samples into liquid nitrogen inside a helium bath cryostat. All experiments were performed at $1.8 \pm 0.1 \mathrm{~K}$, except where indicated.

The experimental setup to measure three pulse-stimulated photon echoes (3PSEs) and 2PEs has been described in great detail elsewhere. ${ }^{13,16}$ The excitation wavelength was $585 \mathrm{~nm}$. Photochemical hole burning of the sample was observed, but on the time scale of a single echo decay measurement (ca. $10 \mathrm{~min}$ ) no corrections were found to be necessary. To minimize any fluence dependence of the echo decays, the excitation pulse energy was held below $5 \mathrm{~nJ}$ for the first two excitation pulses and below $10 \mathrm{~nJ}$ for the third pulse. 2PEs were measured in a three-pulse configuration, i.e., as a 3PSE with the second and third pulse arriving at the sample at the same time. Fluorescence lifetimes were measured on a time-correlated single-photon-counting setup which has also been described elsewhere. ${ }^{17}$

\section{Results and Discussion}

Figure 2 shows decays of the 2PE and of the 3PSE for a waiting time of $3 \mathrm{~ms}$. The decay rate of the $2 \mathrm{PE}$ is twice that of the 


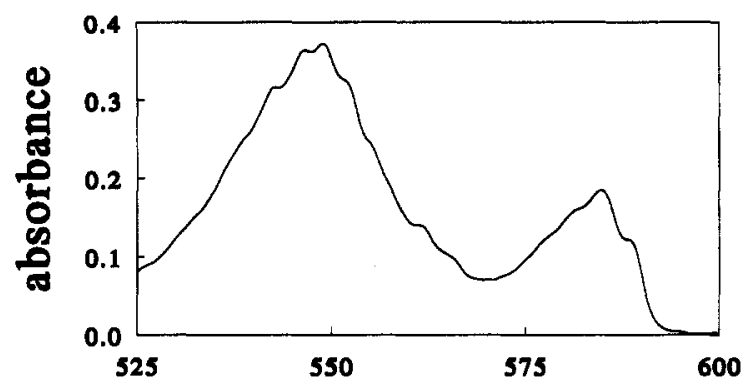

wavelength ( $\mathrm{nm})$

Figure 1. Optical absorption spectrum of $\mathrm{Zn}$-cyt $c$ in a [3/1 vol glycerol/ $10 \mathrm{mM}$ phosphate buffer] host glass at $2.0 \mathrm{~K}$.

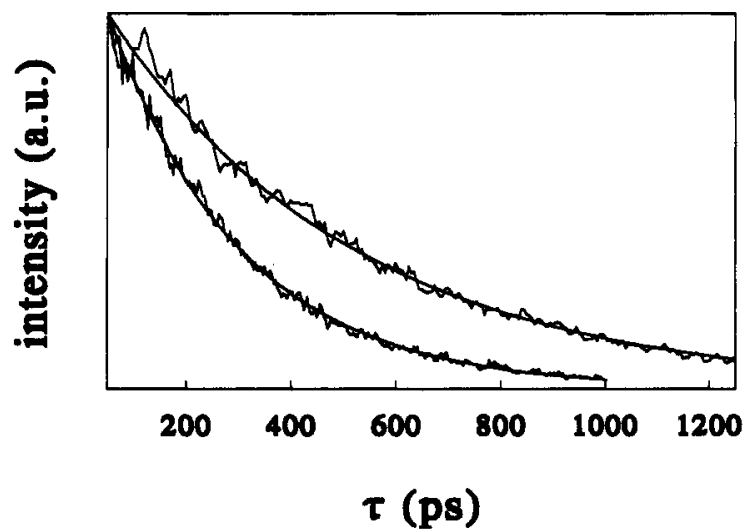

Figure 2. Decays of the 2PE (slow decay) and of a 3PSE with $t_{\mathrm{w}}=3$ $\mathrm{ms}$ (fast decay). The solid lines are single-exponential fits with $T_{2} / 4=$ $477 \mathrm{ps}$ and $T_{2} / 4=256 \mathrm{ps}$, respectively.

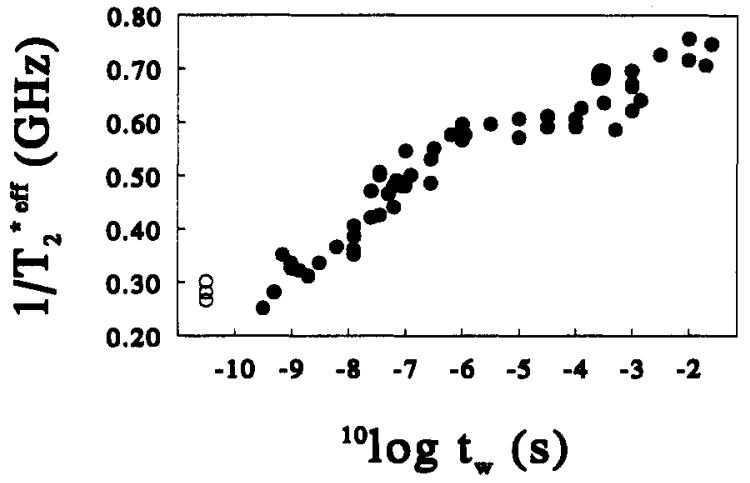

Figure 3. $1 / T_{2}^{*}$ eff vs $t_{\mathrm{W}}$ (closed circles). The open circles are measurements of the $2 \mathrm{PE}$.

lifetime-limited value (vide infra). This indicates that the protein has structural degrees of freedom even at a temperature as low as $1.8 \mathrm{~K}$. Furthermore, the difference in the echo decay rate between the 2PE and the 3PSE with a waiting time of 3-ms signals that these degrees of freedom cover a broad range of time scales. Decays for all waiting times studied in this paper can be fitted reasonably well with a single exponential. The exponential behavior of the 2PE contrasts with the results of Saikan et al. ${ }^{10}$ on PP-myoglobin. At this point, it is difficult to judge whether this difference is caused by differences in the dynamical behavior between both proteins; further studies will be neccesary.

The main result of this study is displayed in Figure 3, which shows the inverse of the effective pure dephasing time, $1 / T_{2}{ }^{*}$ eff, as a function of the waiting time, $t_{\mathrm{w}}$, between the second pulse and the third pulse in the 3PSE experiment. $T_{1}$ (the fluorescence lifetime) was determined to be $1.9 \mathrm{~ns}$. Also shown in Figure 3 are the results of the $2 \mathrm{PE}$. It can be seen that $1 / T_{2}{ }^{*}$ eff initially increases as a function of $t_{\mathrm{W}}$ before reaching a plateau around $t_{\mathrm{W}} \approx 1 \mu \mathrm{s}$. This plateau lasts until $t_{\mathrm{W}} \approx 0.1 \mathrm{~ms}$, after which
$1 / T_{2}{ }^{* e f f}$ starts to increase again. Before analyzing this result in detail, we wish to emphasize that a similar plateau has been observed for $\mathrm{Zn}$-porphin ( $\mathrm{Zn}-\mathrm{P})$ in an ethanol- $d$ glass at $1.5 \mathrm{~K} .^{13}$

In the following we will interpret our experimental data within the TLS model. Using the uncorrelated sudden jump model of $\mathrm{Hu}$ and Walker, ${ }^{18}$ Bai and Fayer ${ }^{19}$ derived for the derivative of the 3PSE-decay rate, $k_{3 \mathrm{PSE}}$, with respect to $t_{\mathrm{W}}$ :

$$
\partial k_{3 \mathrm{PSE}} / \partial t_{\mathrm{W}}=C \int_{0}^{\infty} \mathrm{d} R P(R) R \exp \left[-R t_{\mathrm{w}}\right]
$$

where $P(R)$ is a distribution function of TLS relaxation rates, $R$. $C$ is a constant representative of the strength of the TLSchromophore coupling and the TLS density. Equation 1 is valid only in the long waiting time limit: $t_{W}>10 \tau, \tau$ being the time separation between the first pulse and the second pulse in the 3PSE experiment. From eq 1 it follows that the plateau in Figure 3 corresponds to a gap in $P(R)$ for relaxation rates roughly between $1 \mathrm{MHz}$ and $10 \mathrm{kHz}$. Equation 1 also allows us to fit the data to a particular expression for $P(R)$. Previously, both phenomenological ${ }^{13,20}(P(R) \propto 1 / R)$ and nonphenomenological ${ }^{20,21}$ expressions for $P(R)$ have been used to interpret time-resolved spectral diffusion data. The appearance of a plateau such as in Figure 3 may suggest the existence of two distinct types of TLSs. In this case the experimental data should be described by two distribution functions as opposed to a single distribution with a gap.

We can express the dependence of $k_{3 \mathrm{PSE}}$ on $t_{\mathrm{W}}$ as

$$
k_{3 \mathrm{PSE}}\left(t_{\mathrm{W}}\right)=k_{3 \mathrm{PSE}}\left(t_{\mathrm{W}}{ }^{0}\right)+\int_{t_{\mathrm{w}}{ }^{0}}^{t_{\mathrm{w}}} \mathrm{d} t_{\mathrm{W}}^{\prime} \frac{\partial k_{3 \mathrm{PSE}}}{\partial t_{\mathrm{W}}^{\prime}}
$$

Substitution of eq 1 in eq 2 yields

$$
\begin{array}{r}
k_{3 \mathrm{PSE}}\left(t_{\mathrm{W}}\right)=k_{3 \mathrm{PSE}}\left(t_{\mathrm{W}}{ }^{0}\right)+\int_{0}^{\infty} \mathrm{d} R P(R)\left\{\exp \left[-R t_{\mathrm{W}}^{0}\right]-\right. \\
\left.\exp \left[-R t_{\mathrm{W}}\right]\right\}
\end{array}
$$

The integral in eq 3 can be regarded as a contribution due to spectral diffusion between $t_{\mathrm{w}}{ }^{0}$ and $t_{\mathrm{w}}$. It should be stressed that this approach is valid only when both $t_{\mathrm{w}}{ }^{0}$ and $t_{\mathrm{w}}$ are in the long waiting time limit. Therefore, the use of eq 3 to express $k_{3 \text { PSE- }}$ $\left(t_{\mathrm{W}}\right)$ as the sum of the 2PE decay rate, i.e., $k_{3 \mathrm{PSE}}\left(t_{\mathrm{W}}=0\right)$, and a contribution due to spectral diffusion, as Jankowiak and Small do, ${ }^{21}$ is incorrect.

Parts a and $c$ of Figure 4 show the experimental data of Figure 3 with $t_{\mathrm{w}} \geq 12 \mathrm{~ns}$ together with a fit using eq 3 and the distribution functions displayed in parts $b$ and $d$ of Figure 4, respectively.

The distribution function of Figure $4 b$ is given by

$$
\begin{gathered}
\omega_{1} \text { for } A<\log R \\
P(R) R=0 \text { for } A>\log R>B \\
\omega_{2} \text { for } B>\log R
\end{gathered}
$$

The distribution function of Figure $4 d$ is given by

$$
P(R) R=\sum_{i=1}^{i=2} \frac{c_{i}}{\sigma_{i} \sqrt{2 \pi}} \exp \left[-\left(\frac{\ln \left(R / R_{0, i}\right)}{\sqrt{2} \sigma_{i}}\right)^{2}\right]
$$

Equation 5 comprises two log-normal distributions in the limit of weak TLS-phonon coupling. The log-normal form was proposed by Jankowiak et al. ${ }^{22}$

Figure 4 shows that the use of eq 3 in combination with either one of the distributions given by eqs 4 or 5 gives a reasonable fit to the experimental data with waiting times of $12 \mathrm{~ns}$ and longer. Obviously, eq 3 is insensitive to the exact shape of $P(R)$. Equation 4 might even be regarded as a practical approximation of two physically grounded distributions. Before making an attempt to 

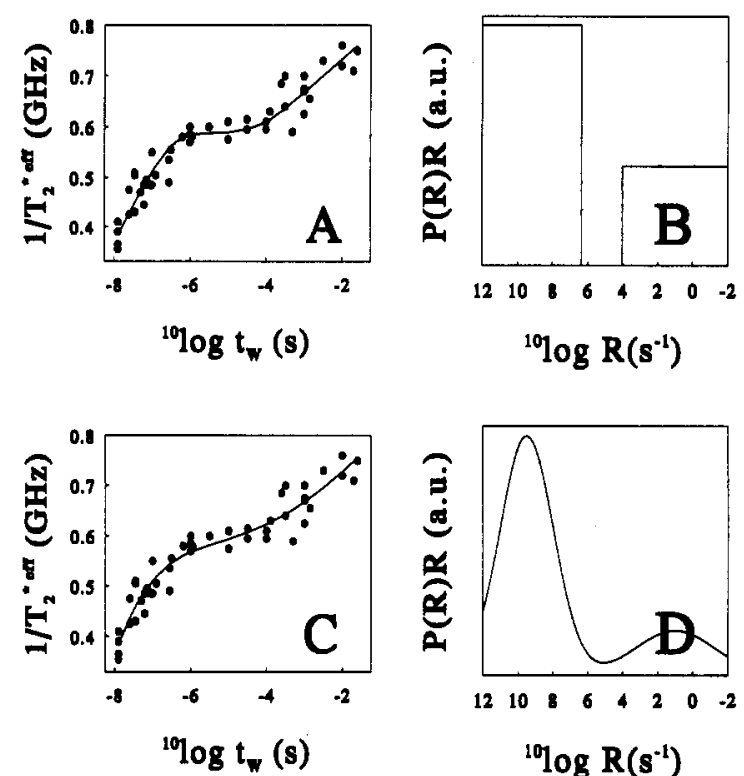

Figure 4. Experimental data of Figure 3 (closed circles) together with a fit (solid line) using the distribution on the right-hand side as discussed in the text. The distribution $B$ is given by eq 4 with $\omega_{1}=67 \mathrm{MHz} ; \omega_{2}$ $=28 \mathrm{MHz} ; A=6.35 ; B=4.0$. The distribution $D$ is given by eq 5 with $R_{0,1}=10^{9.5}\left(\mathrm{~s}^{-1}\right) ; R_{0,2}=10^{1.0}\left(\mathrm{~s}^{-1}\right) ; \sigma_{1}=3.53 ; \sigma_{2}=5.26 ; \mathrm{c}_{1}=1.5 \mathrm{GHz}$ $c_{2}=0.42 \mathrm{GHz}$.

relate these observations to actual physics we wish to make two remarks concerning the properties of an appropriate distribution function. Jankowiak and Small ${ }^{21}$ argued that the faster relaxing type of TLS is intrinsic to the host material and this type of TLS may also be described by a distribution based on strong TLSphonon coupling. This latter distribution exhibits a pronounced tailing toward slower relaxation rates and therefore cannot account for the observed plateau. Another requirement for an appropriate distribution is that besides predicting the waiting time dependence of the 3PSE, it should also account for the decay of the 2PE. This is an important point since both the decay of the $2 \mathrm{PE}$ and the 3PSE for any waiting time are determined by relaxation of TLSs and cannot be interpreted independently. Maynard et al. ${ }^{23}$ showed that an exponential decay of the $2 \mathrm{PE}$ imposes the restriction that $P(R) \propto 1 / R$ for relaxation rates faster than $1 / T_{2}$ and that the TLS-chromophore coupling is dipolar. Littau et al. ${ }^{24}$ derived the following simple relation between the pure dephasing rate obtained from the $2 \mathrm{PE}, 1 / T_{2}{ }^{*}$, and the coupling constant, $\omega$, of the $\omega / R$ distribution:

$$
1 / T_{2}^{*}=3.66 \omega
$$

From Figure $3 \mathrm{~b}$ we find a value of $\omega_{1}$ of $67 \mathrm{MHz}$ which predicts a value of $1 / T_{2}^{*}$ of $0.25 \mathrm{GHz}$. This is in excellent agreement with the measurements of the 2PE (see Figure 3). A log-normal distribution cannot account for both the exponential shape and the rate of the 2PE decay as well as the dependence on $t_{\mathrm{W}}$ of the 3PSE with submicrosecond waiting times.

The previous discussion has shown that the spectral diffusion of the $\mathrm{Zn}$-cyt $c /$ glycerol/water system can be described using the TLS model in combination with a distribution that covers relaxation rates of several orders of magnitude. The important question that still remains unanswered is whether the behavior of this system can in any way be distinguished from that of glasses. A crucial aspect in the interpretation of optical experiments on heme proteins is to what extent the porphyrin is decoupled from the structural parameters (TLSs) of the host glass. On the basis of the differences in general spectral features and temperature cycle induced spectral diffusion between small proteins and glasses, Zollfrank et al. ${ }^{8}$ concluded that the porphyrin in the protein crevice must be decoupled to a large extent from the dynamics of the host glass. To confirm this for our experiment, we would have to perform the same measurement on $\mathrm{Zn-MP}$ in a glycerol/water glass. Unfortunately, Zn-MP does not dissolve in glycerol/water mixtures. Consequently, the possibility that (part of) the observed spectral diffusion results from the dynamics of the host glass cannot be ruled out completely. However, we repeated the experiment on $\mathrm{Zn}$-cyt $\mathrm{c}$ with a different glycerol/water ratio (3/2 volume) to observe identical behavior.

A complicating factor is that previous experiments have shown no such thing as general glasslike behavior in terms of both magnitude and time dependence of spectral diffusion. ${ }^{14}$ As was mentioned earlier, the functional dependence of $1 / T_{2}{ }^{* e f f}$ on $t_{\mathrm{W}}$ of the $\mathrm{Zn}$-cyt $c$ system is similar to that of $\mathrm{Zn}-\mathrm{P}$ in an ethanol-d glass in the sense that for both systems a plateau is observed. However, the magnitude of the spectral diffusion is much larger for the ethanol- $d$ glass, where $1 / T_{2}{ }^{*}$ eff changes from $0.5 \mathrm{GHz}$ for $t_{\mathrm{W}}=0(2 \mathrm{PE})$ to $2 \mathrm{GHz}$ for $t_{\mathrm{W}}=10 \mathrm{~ms}$. Also the plateau seems to extend to longer $t_{\mathrm{W}}\left(\log t_{\mathrm{W}} \approx-2.5\right)$ for the ethanol- $d$ glass. The most important difference with the $\mathrm{Zn}$-cyt $c$ system is that for the latter system the experimental data cannot be described with a hyperbolic distribution function with a gap and a single coupling constant, $\omega$, as is the case for the ethanol- $d$ glass. This strongly suggests that the fast part and the slow part of the distribution represent physically distinguishable TLSs. This view is not completely new. Both Narasimhan et al., ${ }^{25}$ on the basis of hole burning studies of cresyl violet in ethanol glass, and Basché et al., ${ }^{26}$ on the basis of single-molecule spectroscopy on perylene in polyethylene, proposed a clear distinction between the fast and the slow fluctuations. The fast fluctuations result from the interaction of the chromophore with the bulk of the host material. The slow fluctuations correspond to interactions of the chromophore with the solvation shell. The specific interactions of the chromophore with its immediate surroundings are often of an electrostatic nature and barriers for fluctuations are expected to be high and consequently their rates are expected to be slow. We consider the existence of a plateau in Figure 3 as clear evidence for such a theory.

If we accept the existence of two types of TLSs as a general phenomenon, we can bring the time-resolved spectral diffusion of different types of amorphous materials together in a unified picture. Within this picture the appearance of a plateau is not unexpected but merely depends on the parameters that determine the position, shape, and width of the distributions. A plateau is likely to appear when one or both of the distributions is particularly narrow. A narrow distribution of relaxation rates corresponds to a limited number of degrees of structural freedom. In the case of both an ethanol glass and a protein the number of degrees of freedom is limited by the presence of hydrogen bonds. Differences in the magnitude of spectral diffusion can be rationalized in terms of the strength of the TLS-chromophore coupling and of the density of TLSs.

At the end of this discussion we would like to return to the concept of conformational substates. Ansari et al. ${ }^{27}$ proposed that a hierarchy of protein substates exists. The optical absorption spectrum of Zn-cyt c at $2 \mathrm{~K}$, shown in Figure 1, exhibits substantial structure. This structure is probably due to the overlap of several distinct bands with an inhomogeneous width of several tens of wavenumbers. These distinct bands correspond to protein substates of high order in the hierarchy. The spectral fluctuations that cause an increase of the effective homogeneous line width of $0.5 \mathrm{GHz}$ in our experiment are of the order of less than tenth of a wavenumber. In terms of a hierarchy of substates these fluctuations are induced by a low order or substates, differing by very small mean displacements of individual atoms from their equilibrium positions.

As was mentioned earlier, the temperature cycling hole-burning experiments of Zollfrank et al. ${ }^{8}$ on MP-horseradish peroxidase showed a steplike increase of the hole width around $10 \mathrm{~K}$ which 


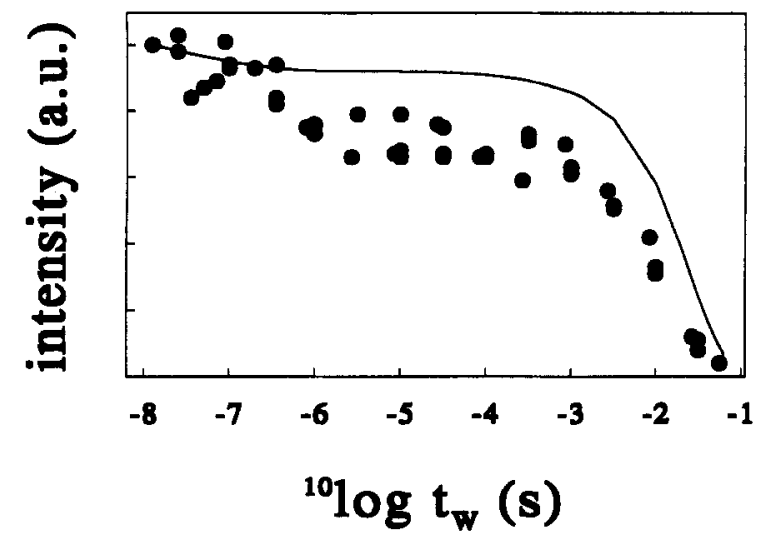

Figure 5. Intensity of the 3PSE at a fixed $\tau$ of $100 \mathrm{ps} v s$. The intensity is plotted relative to the value at $t_{\mathrm{W}}=12 \mathrm{~ns}$. The solid line is a fit as discussed in the text.

they ascribed to a small number, possibly one, of TLSs. Here, we show that a distribution function covering several orders of magnitude of relaxation rates is needed to describe the spectral diffusion at a fixed temperature of $1.8 \mathrm{~K}$. These observations are not neccesarily contradictory. The TLSs Zollfrank et al. are referring to may correspond to a higher order in the hierarchy of substates with barriers that are not surpassable at $1.8 \mathrm{~K}$. Furthermore, the temperature cycling hole burning experiment is sensitive to much slower fluctuations than the long-lived stimulated photon echo.

Figure 5 displays the intensity of the 3PSE at a fixed delay, $\tau$, between the first and the second pulse of $100 \mathrm{ps}$ as a function of $t_{\mathrm{w}}$. The intensity is plotted relative to the intensity at $t_{\mathrm{w}}{ }^{0}=$ $12 \mathrm{~ns}$. At $t_{\mathrm{W}}=12 \mathrm{~ns}$ all the excited-state population has decayed to the ground state, and the echo is solely stimulated from a ground-state grating. The treatment of Hu and Walker ${ }^{18}$ allows us to express the relative echo intensity, $I_{3 \mathrm{PSE}^{\mathrm{rel}}}\left(\tau, t_{\mathrm{W}}\right)$, simply as

$$
I_{3 \mathrm{PSE}}^{\mathrm{rel}}\left(\tau, t_{\mathrm{W}}\right)=\frac{\exp \left[-\tau / T_{3 \mathrm{PSE}}\left(t_{\mathrm{W}}\right)\right]}{\exp \left[-\tau / T_{3 \mathrm{PSE}}\left(t_{\mathrm{W}}^{0}\right)\right]} \frac{\exp \left[-2 t_{\mathrm{W}} / T_{\mathrm{B}}\right]}{\exp \left[-2 t_{\mathrm{W}}^{0} / T_{\mathrm{B}}\right]}
$$

with $T_{3 \mathrm{PSE}}=1 / k_{3 \mathrm{PSE}}$ and $T_{\mathrm{B}}$ the lifetime of the bottleneck state, in this case the triplet state. The solid line in Figure 5 is obtained by calculating $T_{3 \mathrm{PSE}}$ according to eq 3 using the distribution function of Figure $4 \mathrm{~b}$ and a triplet-state lifetime of $50 \mathrm{~ms}$. The fast decay of the echo intensity on a millisecond time scale can be attributed to the decay of the triplet state. The intensity loss on the submicrosecond time scale cannot be accounted for completely using eq 7 . This phenomenon has also been observed for $\mathrm{Zn}-\mathrm{P}$ in glasses and polymers. ${ }^{14}$ However, for these systems the effect was far more pronounced. The anomalous intensity loss may be attributed to a subset of chromophores that experience very large spectral shifts. These shifts are induced by a TLS that is very strongly coupled to the chromophore and that relaxes on a submicrosecond time scale. When these spectral shifts are of the order of the laser bandwidth or larger (several wavenumbers) a loss of echo intensity is expected without affecting the exponential behavior of the echo within the time resolution of the experiment.

Acknowledgment. We gratefully acknowledge Dr. A. A. van Dijk and Prof. G. T. Robillard for their assistance with the sample preparation. We are indebted to F. de Haan for providing us with software for data acquisition and handling. This work was supported by the Netherlands Foundation for Physical Research (FOM) with financial aid from the Netherlands Organization for Scientific Research (NWO).

\section{References and Notes} 1598.

(1) Frauenfelder, H.; Sligar, S. G.; Wolynes, P. G. Science 1991, 254,

(2) Frauenfelder, H.; Petsko, G. A.; Tsernoglou, D. Nature (London) 1979, 280, 558. 25,1 .

(3) Anderson, P. W.; Halperin, B. I.; Varma, C. M. Philos. Mag. 1972,

(4) Philips, W. A. J. Low Temp. Phys. 1972, 7, 351

(5) Narasimhan, L. R.; Littau, K. A.; Pack, D. W.; Bai, Y. S.; Elschner, A.; Fayer, M. D. Chem. Rev. 1990, 90, 439.

(6) Boxer, S. G.; Gottfried, D. S.; Lockhart, D. J.; Middendorf, T. R. J. Chem. Phys. 1987, 86, 2439 4388 .

(7) Koloczek, H.; Fidy, J.; Vanderkooi, J. M. J. Chem. Phys. 1987, 87,

(8) Zollfrank, J.; Friedrich, J.; Vanderkooi, J. M.; Fidy, J. Biophys. J. $1991,59,305$.

(9) Zollfrank, J.; Friedrich, J.; Vanderkooi, J. M.; Fidy, J. J. Chem. Phys. 1991, 95, 3143.

(10) Saikan, S.; Lin, J. W.-I.; Nemoto, H. Phys. Rev. B 1992, 46, 11125.

(11) Gafert, J.; Friedrich, J.; Parak, F. J. Chem. Phys. 1993, 99, 2478.

(12) Morsink, J. B.; Hesselink, W. H.; Wiersma, D. A. Chem. Phys. 1982. $71,289$.

(13) Meijers, H. C.; Wiersma, D. A. Phys. Rev. Lett. 1992, 68, 381

(14) Meijers, H. C. Thesis, University of Groningen, 1994.

(15) Vanderkooi, J. M.; Adar, F.; Erecifiska, M. Eur. J. Biochem. 1976, 64,381 .

(16) Meijers, H. C.; Wiersma, D. A. Chem. Phys. Lett. 1991, 81, 312.

(17) De Boer, S.; Wiersma, D. A. Chem. Phys. Lett. 1990, 165, 45.

(18) Hu, P.; Walker, L. R. Phys. Rev. B 1978, 18, 1300.

(19) Bai, Y. S.; Fayer, M. D. Phys. Rev. B 1989, 39, 11066

(20) Littau, K. A.; Fayer, M. D. Chem. Phys. Lett. 1991, 176, 551.

(21) Jankowiak, R.; Small, G. J. Phys. Rev. B 1993, 47, 14805.

(22) Jankowiak, R.; Small, G. J.; Athreya, K. B. J.Phys. Chem. 1986, $90,3896$.

(23) Maynard, R. D.; Rammal, R.; Suchail, R. J. Phys. (Paris) 1980, 41, L291, L614.

(24) Littau, K. A.; Dugan, M. A.; Chen, S.; Fayer, M. D. J. Chem. Phys. $1992,96,3484$

(25) Narasimhan, L. R.; Pack, D. W.; Fayer, M. D. Chem. Phys. Lett. 1988, $152,287$.

(26) Basché, Th.; Ambrose, W. P.; Moerner, W. E. J. Opt. Soc. Am. B $1992,5,829$.

(27) Ansari, A.; Berendzen, J.; Bowne, S. F.; Frauenfelder, H.; Iben, I. E. T.; Sauke, T. B.; Shyamsunder, E.; Young, R. D. Proc. Natl. Acad. Sci. U.S.A. 1985, 82, 5000 . 